\title{
Compensatory role of inducible annexin A2 for impaired biliary epithelial anion-exchange activity of inflammatory cholangiopathy
}

\author{
Osamu Kido ${ }^{1}$, Koji Fukushima ${ }^{1}$, Yoshiyuki Ueno ${ }^{1}$, Jun Inoue ${ }^{1}$, Douglas M Jefferson ${ }^{2}$ and Tooru Shimosegawa ${ }^{1}$
}

The peribiliary inflammation of cholangiopathy affects the physiological properties of biliary epithelial cells (cholangiocyte), including bicarbonate-rich ductular secretion. We revealed the upregulation of annexin A2 (ANXA2) in cholangiocytes in primary biliary cirrhosis (PBC) by a proteomics approach and evaluated its physiological significance. Global protein expression profiles of a normal human cholangiocyte line (H69) in response to interferon- $\gamma$ (IFN $\gamma$ ) were obtained by two-dimensional electrophoresis followed by MALDI-TOF-MS. Histological expression patterns of the identified molecules in PBC liver were confirmed by immunostaining. H69 cells stably transfected with doxycyclin-inducible ANXA2 were subjected to physiological evaluation. Recovery of the intracellular $\mathrm{pH}$ after acute alkalinization was measured consecutively by a pH indicator with a specific inhibitor of anion exchanger (AE), 4,4'-diisothiocyanatostilbene-2,2'disulfonic acid (DIDS). Protein kinase-C (PKC) activation was measured by PepTag Assay and immunoblotting. Twenty spots that included ANXA2 were identified as IFN $\gamma$-responsive molecules. Cholangiocytes of PBC liver were decorated by the unique membranous overexpression of ANXA2. Apical ANXA2 of small ducts of PBC was directly correlated with the clinical cholestatic markers and transaminases. Controlled induction of ANXA2 resulted in significant increase of the DIDS-inhibitory fraction of AE activity of $\mathrm{H69}$, which was accompanied by modulation of PKC activity. We, therefore, identified ANXA2 as an IFN $\gamma$-inducible gene in cholangiocytes that could serve as a potential histological marker of inflammatory cholangiopathy, including PBC. We conclude that inducible ANXA2 expression in cholangiocytes may play a compensatory role for the impaired $A E$ activity of cholangiocytes in $\mathrm{PBC}$ in terms of bicarbonate-rich ductular secretion and bile formation through modulation of the PKC activity.

Laboratory Investigation (2009) 89, 1374-1386; doi:10.1038/labinvest.2009.105; published online 12 October 2009

KEYWORDS: primary biliary cirrhosis (PBC); proteomics; human cholangiocyte; Tet-On sysem; protein kinase-C (PKC)

Primary biliary cirrhosis $(\mathrm{PBC})$ is characterized by chronic bile duct injury and cholestasis in the early disease stage, and its etio-pathogenesis is still unrevealed. Chronic inflammatory cells, especially $\mathrm{CD} 8^{-}$and $\mathrm{CD} 4^{-}$lymphocytes that infiltrate around the bile ducts, are considered to be associated with the progression of bile duct injury and cholestasis in PBC through membranous death receptors and cytokine receptors. ${ }^{1-4}$ Proinflammatory cytokines secreted by these inflammatory cells, such as interleukin (IL)-6, interferon- $\gamma($ IFN $\gamma)$, tumor necrosis factor- $\alpha$ and IL-1, would also cause cholestasis by means of impairment of the ductular secretion through their inhibitory effect on cyclic adenosine monophosphate (cAMP) formation, anion exchanger (AE) activity and cAMP-dependent $\mathrm{Cl}^{-}$efflux. ${ }^{5}$ These findings may be related to the manifestation of cholestasis in the initial disease stage without any evident features of chronic non-suppurative destructive cholangitis. The global profiling technique is regarded as a powerful methodology for the framing of a hypothesis. In the current study, we employed proteomics for the profiling of biliary epithelial cells, cholangiocytes, in terms of Th1 cytokine responses in order to screen for novel biomarkers of inflammatory cholangiopathy and to provide insight into the mechanism of its pathophysiology.

Annexin-A2 (ANXA2), one of the candidate molecules based on the results of proteomics applied to H69 cells, is a $\mathrm{Ca}^{2+}$ - and acidic phospholipid-binding protein involved in many cellular processes. ANXA2, which can associate with

\footnotetext{
${ }^{1}$ Division of Gastroenterology, Tohoku University Graduate School of Medicine, Sendai, Japan and ${ }^{2}$ Department of Physiology, Tufts University, Boston, MA, USA Correspondence: Dr Y Ueno, MD, PhD, Division of Gastroenterology, Tohoku University Graduate School of Medicine, 1-1 Seiryo Sendai Aoba, Sendai, Miyagi 980-8574, Japan. E-mail: yueno@mail.tains.tohoku.ac.jp
}

Received 25 September 2008; revised 21 July 2009; accepted 18 August 2009 
caveolae, has been shown to form a lipid-protein complex and cholesteryl esters that seem to be involved in the internalization/transport of cholesteryl esters from caveolae to internal membranes. ${ }^{6-10}$ ANXA2 is also required for transport of secretory vesicles in adipocytes and exocytic vesicles in polarized epithelial cells. ${ }^{11}$ It has been investigated extensively in the field of cardiovascular system and has various roles, including coagulopathy. ${ }^{12}$ In contrast to cardiovascular system, the role of ANXA2 in the hepato-biliary systems is not well understood. In terms of alcoholic liver diseases, an increase of parenchymal ANXA2 expression and a fibrinolytic role in the liver has been proposed. ${ }^{13}$

Apical expression of $\mathrm{Cl}^{-} / \mathrm{HCO}^{-}$anion exchanger-2 (AE2: SLC4A2 as an official gene symbol) plays a pivotal role in the regulation of the intracellular $\mathrm{pH}\left(\mathrm{pH}_{\mathrm{i}}\right)^{14}$ in conjunction with the biliary $\mathrm{HCO}^{-}$secretion and bile formation by cholangiocytes. ${ }^{15}$ Faint expression of AE2 in the cholangiocytes of $\mathrm{PBC}$ livers ${ }^{16}$ may suggest some potential roles of $\mathrm{AE}$ in the development of $\mathrm{PBC}$, which is further supported by cholangiopathy characterized by portal inflammation and bile duct injury observed in AE-deficient mice. ${ }^{17}$ Therefore, in the current study, we focused on the pathophysiological relevance of ANXA2 to biliary excretion in terms of anion-exchange activity.

\section{MATERIALS AND METHODS Materials}

The reagents for the cell culture, electrophoresis and mass spectrometry were purchased from Wako Pure Chemical Industries Ltd. (Japan), excluding Bio-Lyte ${ }^{\mathbb{R}} 3 / 10$ carrier ampholyte, ReadyStrip IPGStrips, PROTEAN IEF Cell, Tris/ $\mathrm{HCl}(\mathrm{pH} 8.8$ ), ReadyGel J and flamingo gel stain (Bio-Rad, Hercules, CA, USA), Typhoon Variable Mode Imager, ImageMaster 2DPlatinum and Ettan SpotPicker (GE Healthcare, Piscataway, NJ, USA), $\alpha$-cyano-4-hydroxy-cinnamic acid, doxycycline (DOX), 4,4'-diisothiocyanatostilbene-2,2'-disulfonic acid (DIDS), IFN $\gamma$ (Pierce Endogen, Rockford, IL, USA), adenosine $5^{\prime}$-[ $\gamma$ thio]triphosphate tetralithium salt (ATP $\gamma$ S), 3-isobutyl-1-methylxanthine (IBMX), propionic acid and Krebs-Ringer bicarbonate (KRB) (Sigma-Aldrich, St Louis, MO, USA), Voyager-DE STR BiospectrometryWorkstation (Applied Biosystems, Foster City, CA, USA), 3'-OAcetyl-2',7'-bis(carboxyethyl)-4,5-carboxyfluorescein, diacetoxymethyl ester (BCECF-AM) (Molecular Probes, Eugene, OR, USA), FluoroskanAscent (MTX Lab Systems, Vienna, Virginia), phorbol-12-myristate-13-acetate (PMA), bisindolylmaleimide-I (Go6850, BIS) (Calbiochem, Darmstadt, Germany) and forskolin (Biomol, Plymouth Meeting, PA, USA). Anti-ANXA2 (monoclonal antibody (AB); BD Bioscience, San Jose, CA, USA), anti-AE2 (polyclonal AB; Santa Cruz Biotechnology, Santa Cruz, CA, USA), anticyclin-I and anti-Epac1 (polyclonal AB; GeneTex Inc., San Antonio, TX, USA), anti-enolase-1 (monoclonal AB; Abnova Corporation, Taipei, Taiwan), anti-Hsp70 (monoclonal AB; Stressgen, Ann Arbor, MI, USA), anti- $\beta$-actin (monoclonal
AB; Sigma), the phospho-protein kinase-C (PKC) Antibody Sampler kit (polyclonal AB; Cell Signaling, Danvers, MA, USA), N-Histofine Simple Stain MAXPO (NICHIREI BIOSCIENCES INC., Tokyo, Japan), VECTOR NovarRed (VECTOR, Burlingame, CA, USA) were used for immunoblotting and immunostaining. The Alexa $^{-}$-labeled secondary antibodies for immunofluorescence were purchased from Molecular Probes. The materials for construction of expression vectors and transfection experiments were all purchased from Takara Bio Inc. (Japan), except for Tet-On System (Clontech, Mountain View, CA, USA), RNAeasy kit, ANXA2 siRNA, All Stars Negative Control siRNA (Qiagen, the Netherlands), pT7Blue T vector (Novagen Inc., Madison, WI, USA), TransIT ${ }^{\mathbb{R}}$-LT1 Transfection Reagent (Mirus, Madison, WI, USA), G418 (Geneticin) and hygromycin (Invitrogen Corporation, Carlsbad, CA, USA). PepTag Assay for Non-Radioactive Detection of Protein Kinase C or cAMP-Dependent Protein Kinase (PepTag Assay) was purchased from Promega Corporation (Madison, WI, USA).

\section{Methods \\ Cell culture and Sample preparation for 2-dimensional electrophoresis}

The H69 human cholangiocyte line was cultured in Dulbecco's Modified Eagle's Medium ${ }^{18}$ with/without IFN $\gamma$ at a concentration of $1000 \mathrm{IU} / \mathrm{ml}$ for $24 \mathrm{~h}$ at $37^{\circ} \mathrm{C}$ in $\mathrm{CO}_{2}$ incubators. Whole-cell lysates were prepared for two-dimensional electrophoresis (2DE) in the degenerate extraction buffer consisting of $8 \mathrm{M}$ urea, $2 \%$ 3-[(3-cholamidopropyl)dimethylammonio]propanesulfonic acid (CHAPS), $50 \mathrm{mM}$ dithiothreitol (DTT) and 0.2\% Bio-Lyte3/10 carrier ampholyte.

\section{DGE and protein profiling}

A 75 -g weight of each sample was loaded on the ReadyStrip IPGStrips $(7 \mathrm{~cm}, \mathrm{pH}$ 5-8). Isoelectric Focusing was performed under conditions recommended by the manufacturer, briefly at $250 \mathrm{~V}$ for $20 \mathrm{~min}$, then voltage gradually shifted to $4,000 \mathrm{~V}$ for $2 \mathrm{~h}$, followed by $4,000 \mathrm{~V}$ for $10 \mathrm{~h}$, finally $500 \mathrm{~V}$ for $24 \mathrm{~h}$ using PROTEAN IEF Cell. IPGStrips were equilibrated twice in sodium dodecyl sulfate (SDS)-containing buffers consisting of $6 \mathrm{M}$ urea, $2 \%$ SDS, $0.05 \mathrm{M}$ Tris/ $\mathrm{HCl}(\mathrm{pH} 8.8)$, $20 \%$ glycerol, DTT for the first step and iodoacetamide for the second step. Then the strips were placed onto the top of ReadyGel J (5-20\%) for 2D SDS-PAGE. Separated proteins were visualized using the Flamingo fluorescent gel stain and the Typhoon Variable Mode Imager. Corresponding spots of the IFN $\gamma$-stimulated and control samples were automatically determined by the ImageMaster 2DPlatinum ver. 5.0. Spots with fluorescence intensity that changed significantly in comparison with the control were automatically determined by the ImageMaster 2DPlatinum ver. 5.0 and excised by Ettan SpotPicker. After succeeding processes of in-gel digestion, desalting and mixture with the matrix ( $\alpha$-cyano-4-hydroxycinnamic acid), matrix-assisted laser desorption/ionization time-of-flight mass spectrometry (MALDI-TOF-MS) was 
performed with the Voyager-DE STR BiospectrometryWorkstation. Each unique mass spectrum was applied to database search for lining up of the candidates. Identical proteins were determined subsequently by the combination of probability, $\mathrm{pI}$ and molecular weight.

\section{Immunohistochemistry, immunoblotting and immunofluorescence analysis}

Under the approval of the institutional review board, liver specimens were obtained from 20 PBC patients, 20 chronic hepatitis-C $(\mathrm{CHC})$ patients as disease controls and 10 donors for liver transplantation as normal controls. The usage of these specimens was approved by Institutional Review Board (2006-59) along with the written informed consent from the patients. The samples were fixed immediately in $4 \%$ paraformaldehyde (PFA) and embedded in paraffin. For immunohistochemistry, after overnight incubation with the specific antibodies and succeeding incubation with the corresponding peroxidase-labeled secondary antibodies, the expressions of the target molecules were visualized according to the manufacturer's protocol. Histological stages of $\mathrm{PBC}$ were determined according to Scheuer's criteria. ${ }^{19}$ ANXA2 expression in cholangiocytes was classified into three grades and evaluated by two independent pathologists. Electrophoresis and immunoblotting were performed according to the standard protocol of chemiluminiscence under degenerative condition. For immunofluorescence, the cells at subconfluency cultured in a glass-bottomed dish were fixed in $4 \%$ PFA for $1 \mathrm{~h}$ and permeabilized by $0.1 \%$ Triton-X. Fluorescence was observed by confocal microscopy (TE-2000; Nikon, Tokyo, Japan).

\section{Tet-On expression vectors and stable transfection}

The Tet-On vector for DOX inducible ANXA2 expression was constructed as follows: ${ }^{20}$ The coding region of human ANXA2 was obtained by polymerase chain reaction (PCR) from cDNA derived from total RNA of H69 through reverse transcription and subsequently cloned into the pT7Blue $T$ vector. The primers were designed based on the reference sequence (gi: 50845387). The cloned ANXA2 was inserted at SalI-BamHI site of pTRE2Hyg (pTRE2-ANXA2). For preconditioning, H69 cells were stably transfected with pTet-On, which contains DOX-inducible trans-acting element, and subcloned after the 4 weeks of the G418 selection step. Suitable clones were selected by luciferase reporter assay with the DOX-inducible luciferase vector, pTRE2Hyg-Luc. The selected H69 clones were stably transfected with pTRE2ANXA2 and subcloned through the hygromycin selection process. Final clones (H69-anxa2) were examined for the DOX-inducible ANXA2 synthesis by immunoblotting.

\section{Suppression of ANXA2's expression by siRNA}

Shortly before transfection, H69 cells were seeded at a concentration of $2 \times 10^{4}$ cells per well in 96-well plates and $2.5 \times 10^{5}$ cells per well in six-well plates. ANXA2 siRNA and negative control siRNA were transfected at a concentration of $5 \mathrm{nM}$ for $24 \mathrm{~h}$. The amount of expression of ANXA2 was evaluated by western blotting for the determination of the suppressive efficacy of the siRNA.

\section{Measurement of AE activity}

The H69-anxa2 cells were assessed for their AE activity by measuring the recovery of $\mathrm{pH}_{\mathrm{i}}$ from acute alkalization according to the established method. ${ }^{21}$ In brief, for $\mathrm{pH}_{\mathrm{i}}$ measurement, the cell culture medium was substituted with $\mathrm{KRB}$ and KRB-propionate buffers loaded with $10 \mu \mathrm{M}$ of BCECF-AM, and fluorescence intensity was consecutively measured by FluoroskanAscent at $488 \mathrm{~nm}$ for excitation and at $535 \mathrm{~nm}$ for emission. Rates of $\mathrm{pH}_{\mathrm{i}}$ change $\left(\mathrm{dpH}_{\mathrm{i}} / \mathrm{dt}\right)$ were compared under the following experimental conditions: preincubation with an $\mathrm{AE}$ inhibitor, DIDS, at a concentration of $1 \mathrm{mM}$, in the presence of the following agents under the indicated conditions of concentrations and incubation periods: PMA (PKC activator, $50 \mathrm{nM}, 20 \mathrm{~min}$ ), BIS (pan-PKC inhibitor, $1 \mu \mathrm{M}, 10 \mathrm{~min}), \operatorname{ATP} \gamma \mathrm{S}(100 \mu \mathrm{M}, 10 \mathrm{~min})$, forskolin $(100 \mu \mathrm{M} 15 \mathrm{~min})$ with $\mathrm{IBMX}(0.5 \mathrm{mM}, 20 \mathrm{~min})$.

\section{PepTag Assay for non-radioactive detection of PKC activity}

For evaluation of PKC activity, confluent monolayers of H69 cells grown on dishes preincubated in PMA and/or ATP $\gamma \mathrm{S}$ were rinsed twice in ice-cold phosphate-buffered saline and harvested by scraping on ice in $500 \mu \mathrm{l}$ of PKC extraction buffer according to the manufacturer's manual. The cells were lysed by mild sonication in the buffer and submitted to the PepTag Assay.

PKCs are maturated by phosphorylation of multiple serine/threonine sites and further activated by diacylglycerol or its analogues. PepTag Assay is designed for quantification of the actual whole PKC activity with the usage of fluorescent peptide as the specific substrate for PKCs. Activated forms of PKCs change the net charge of the substrate from positive to negative, thereby allowing the phosphorylated and nonphosphorylated substrate to be separated by agarose gel electrophoresis. Cell lysates with the equivalent protein concentration were incubated with the PKC reaction mixture at $30^{\circ} \mathrm{C}$ for $30 \mathrm{~min}$. The reactions were stopped by placing the tubes on a $95^{\circ} \mathrm{C}$ heating block. After adding $80 \%$ glycerol, the samples were electrophoresed in $0.8 \%$ agarose gel for $15 \mathrm{~min}$ at $100 \mathrm{~V}$ and visualized by UV light.

\section{Statistics}

Results were analyzed by paired $t$-test, Mann-Whitney's $U$-test, Kruskal-Wallis test with Bonferroni correction or Spearman's correlation. $P$ values less than 0.05 were considered statistically significant.

\section{RESULTS}

\section{Proteomics}

Three hundred and seventy spots among the 1400 spots detected by 2DGE matched between IFN $\gamma$-treated and 


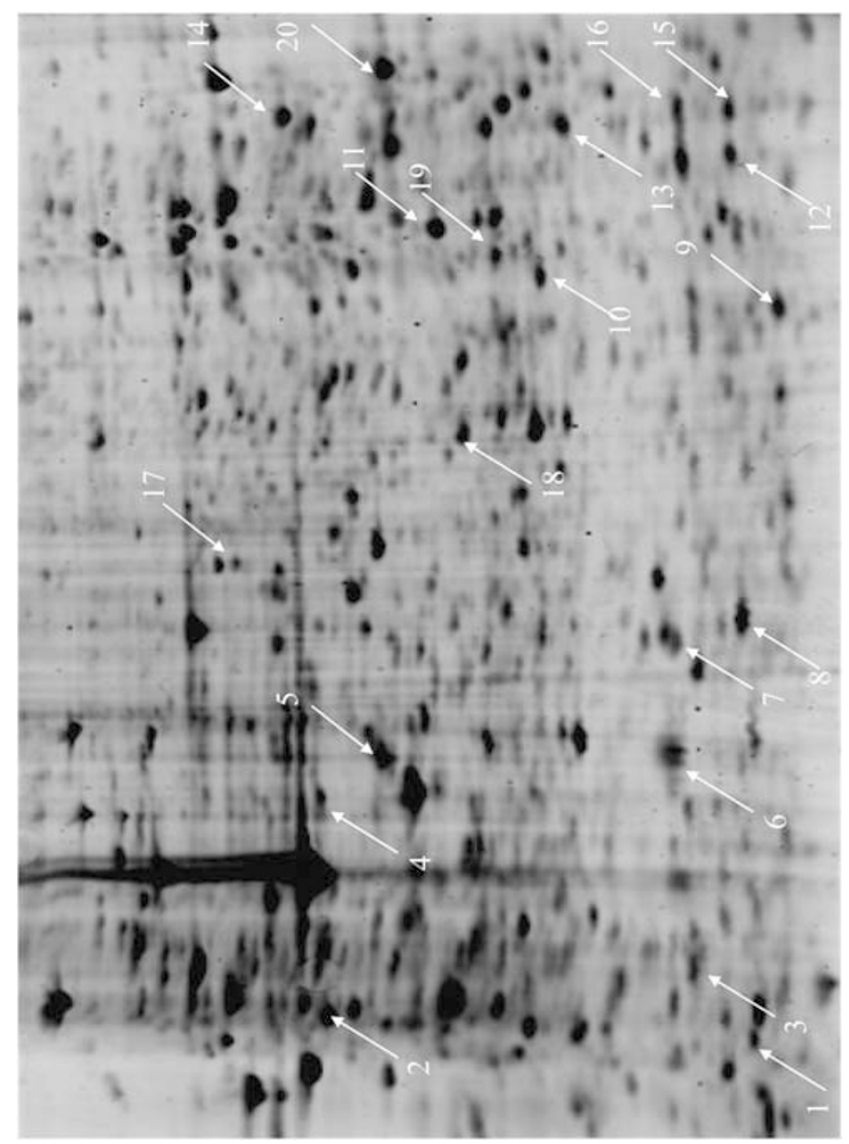

Figure 1 A representative 2DGE image for expression profiling of $\mathrm{H} 69$ cells treated with IFN $\gamma$ is shown. The fluorescence intensities of the 20 spots indicated by the arrows changed significantly in comparison with the control. The intensities for the 16 spots numbered 1 to 16 increased and the four spots numbered 17 to 20 decreased, respectively. Paired $t$-test was applied to triplicate data sets analysis.

IFN $\gamma$-untreated groups of the $\mathrm{H} 69$ cells. The fluorescence intensities of 16 spots (numbers 1 to 16) treated with IFN $\gamma$ were significantly increased compared with that of the untreated spots, and the fluorescence intensities of four spots (numbers 17 to 20 ) were significantly decreased (Figure 1). Proteins identified by MALDI-TOF-MS and peptide mass fingerprinting (PMF) are listed in Table 1.

\section{Cellular and Subcellular ANXA2 Expression of the PBC Liver}

Among these inflammation-related molecules, we focused on the IFN $\gamma$-inducible molecules as candidates of cholangiopathy. Five of 16 molecules were evaluated for expression in the liver specimens by immunohistochemistry (Figures 2 and 3). ANXA2 showed a clear and unique expression pattern in the liver, which was characterized by almost exclusive expression in the cholangiocytes of normal livers as well as parenchymal expression especially in the periportal area of $\mathrm{PBC}$ or $\mathrm{CHC}$ liver with infiltration of inflammatory cells. In accordance with the in vitro results, the expression of ANXA2 in cholangiocytes was augmented in PBC liver that was accompanied by peribiliary inflammation (Figure 3 ). Other molecules detected by TOF-MS were evaluated with regard to cyclin-I, EPAC1, enolase-1 and HSP70 by immunohistochemistry (Figure 2). Cyclin-I was expressed almost exclusively in hepatocytes both in PBC and normal liver. EPAC1 and enolase-1 were expressed equally in biliary epithelial cells of PBC and normal liver. The expression of HSP70 was not clear by the usual immunohistochemistry in these liver specimens. ANXA2 was subcellularly distributed mainly membranously and partly in the cytosol of cholangiocytes in the PBC liver (Figure 3e-g). Especially, its apical expression was unique and other physiological roles of this molecule other than those coagulation-related were speculated. With regard to PBC livers, subcellular localization of ANXA2 to the apical membrane of cholangiocyte was positively correlated with the clinical markers of cholestasis and liver injury such as the serum concentration of alkaline phosphatase, $\gamma$-glutamyltranspeptidase, aspartate aminotransferase (AST), alanine aminotransferase (ALT) and total cholesterol (Table 2). Thereby, its expression was likely to be related to the cholestatic status.

\section{Effect of Controlled Induction of Anxa2 to AE Activity}

To investigate the significance of ANXA2 expression in cholangiocytes, we constructed systems for the controlled induction of ANXA2 in H69 cells under the Tet-On system. We speculated that the apical localization of ANXA2 in correlation to cholestasis might have a close relation to the $\mathrm{AE}$ activity, which was involved in bile formation by the apical domain of cholangiocytes. AE activity was assessed in the ordinary manner, by measurement of DIDS-sensitive intracellular $\mathrm{pH}$ recovery from acute intracellular alkalization (Figure 4). Induction of ANXA2 under DOX trans-activating promoter led to a 2.5-3fold increase of the $\mathrm{pH}_{\mathrm{i}}$ recovery, which was sensitive to DIDS (Figure 4a). IFN $\gamma$ and ATP $\gamma S$ supplemented in the culture media of the cells attenuated the $\mathrm{AE}$ activity, which was partly restored by induction of ANXA2 (Figure 4b, c, d). In contrast, PMA also reduced the AE activity, which was not rescued by ANXA2 expression. BIS and forskolin did not alter the AE activity, regardless of the status of ANXA2. Therefore, ANXA2 was considered to have contributed to the restoration of impaired AE activity probably through some mechanisms that involve PKC. In contrast, ANXA2 expression and $\mathrm{pH}_{\mathrm{i}}$ recovery were significantly decreased simultaneously against the negative control by ANXA2 siRNA to $46.9 \%$ and $57.3 \%$, respectively (Figure 4e-g).

\section{AE2 Expression in $\mathrm{H} 69$ Cells}

The AE activity is mainly assumed to occur by AE2 expression in cholangiocytes. ${ }^{15,22}$ To test the hypothetical ANXA2AE2 pathway, we evaluated the AE2 status in H69. Immunofluorescence and immunoblotting revealed that incubation with IFN $\gamma$ or induction of ANXA2 did not alter 
Table 1 Expression profiling by MALDI-TOF-MS and peptide-mass finger printing

\begin{tabular}{|c|c|c|c|}
\hline Spot number & Gene name & GenBank accession number & Gene ontology \\
\hline 1 & DGCR8 & gi: 50415809 & Transcription \\
\hline 3 & Amilorid-sensitive cation channel 3 & gi: 9998946 & Transport \\
\hline 4 & STAT6 & gi: 58737047 & Transcription \\
\hline 6 & Tryptophanyl-tRNA synthetase & gi:47419918 & Negative control of cell proliferation. \\
\hline 7 & Lines homolog & gi: 95007028 & Transcription \\
\hline 9 & EPAC1 & gi: 32171400 & Cell proliferation \\
\hline 10 & Tyrosine-protein kinase BTK isoform52 & gi: 63147587 & Apoptosis \\
\hline 11 & Annexin A2 & gi: 18088908 & \\
\hline 15 & Cyclin-I & gi: 54696900 & Cyclin family \\
\hline 16 & CLCA homolog & gi: 4572289 & Transport \\
\hline 17 & HNRPH1 & gi: 48145673 & Transcription \\
\hline 18 & C-type lectin superfamily-4 & gi: 38348296 & Cell adhesion \\
\hline 19 & Triosephosphate isomerase-1 & gi: 17389815 & Fatty-acid biosynthetase \\
\hline 20 & Myo-inositol monophospatase-A3 & gi: 17389522 & Hydrolase \\
\hline
\end{tabular}

Abbreviations: MALDI-TOF-MS, matrix-assisted laser desorption/ionization time-of-flight mass spectrometry.

either the amount of expression or subcellular localization, that is, to the cell-surface membrane, of AE2 in polarized H69 cells (Figure $5 \mathrm{a}$ and $\mathrm{b}$ ). Therefore, it was speculated that inducible ANXA2 might restore the AE activity that was impaired under the inflammatory conditions through mechanisms other than changes in the expression or the subcellular localization in biliary epithelial cells or cholangiocytes.

\section{AE Activity, ANXA2 and Involvement of PKC Pathway}

The AE activity was previously reported to be affected by PKC through phosphorylation of the serine/threonine residue of $\mathrm{AE}^{23}$ Similar to PKC, ANXA2 is a calcium-dependent and membrane-associated molecule, which led us to hypothesize that PKC might be involved in the restoration of AE activity by ANXA2. Actually, PMA decreased the AE activity in H69 cells (Figure 4c), in concordance with the redistribution of AE2 protein from the plasma membrane to the intracellular space (Figure 5b). Consistent with our hypothesis, the induction of ANXA2 reduced the PKC activity, as revealed by PepTag Assay (Figure 6a) and immunoblotting (Figure $6 \mathrm{~b}$ and $\mathrm{c}$ ). The extracellular ATP level unexpectedly decreased the AE activity and the induction of ANXA2 partially recovered the diminished AE activity (Figure 4d), which was accompanied by reduction of PKC activity (Figure 6a). Especially, reduction of maturated nPKC was evident compared with that of the other subtypes of PKC (Figure 6b, c).

\section{DISCUSSION}

In the current research, we proposed 20 molecules as candidates, which might play roles in the development of cholangiopathy. ${ }^{24-37}$

The heat-shock $70-\mathrm{kDa}$ protein (HSP70) is a member of HSPs, which are molecular chaperones that control protein folding and prevent aggregation. ${ }^{38}$ Axonemal dynein heavy chain-7 is a force-generating protein of respiratory cilia ${ }^{39}$ and dynein is supposed to facilitate the trafficking of AQP1containing vesicles in cholangiocytes. ${ }^{40}$ The exchange factor directly activated by cAMP-1 (EPAC1) is a guanine nucleotide-exchange factor and a second messenger of cAMP that leads to cell proliferation. ${ }^{41}$ Enolase-1, together with its role in glycolysis, plays a role in various processes such as growth control, hypoxia tolerance and allergic responses, and may also function in the fibrinolytic system as a receptor and activator of plasminogen on the cell surface. ${ }^{42}$ The expression of enolase-1 is assumed to be involved in cholangiocytes destruction and relevant to the disease progression in PBC. ${ }^{43}$

ANXA2 is a membranous acidic phospholipid-binding protein that contains putative binding sites for $\mathrm{Ca}^{2+}$, and is involved in many cellular processes. ${ }^{44,45}$ It undergoes $\mathrm{Ca}^{2+}$ mediated membrane bridging and has been demonstrated to 
a

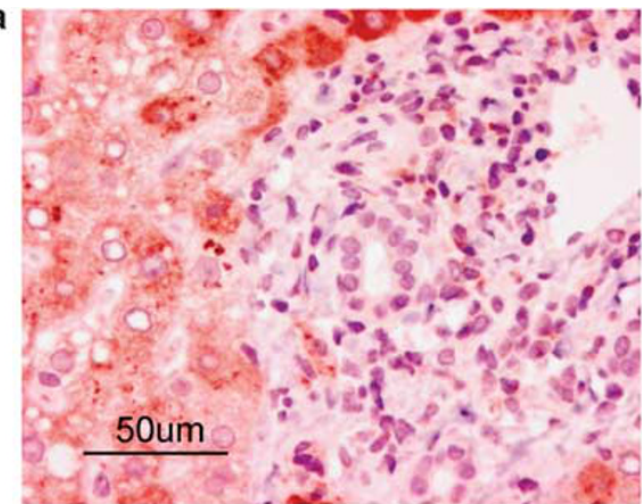

C

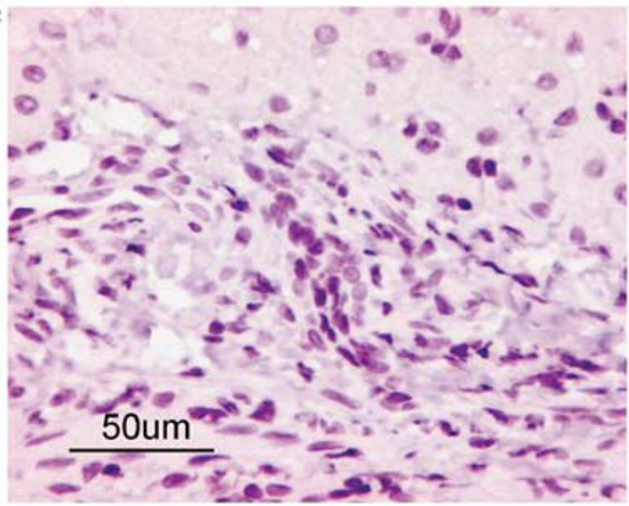

e

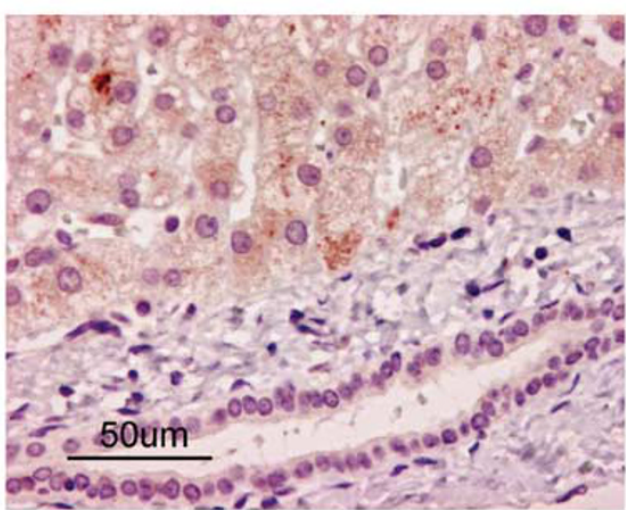

g

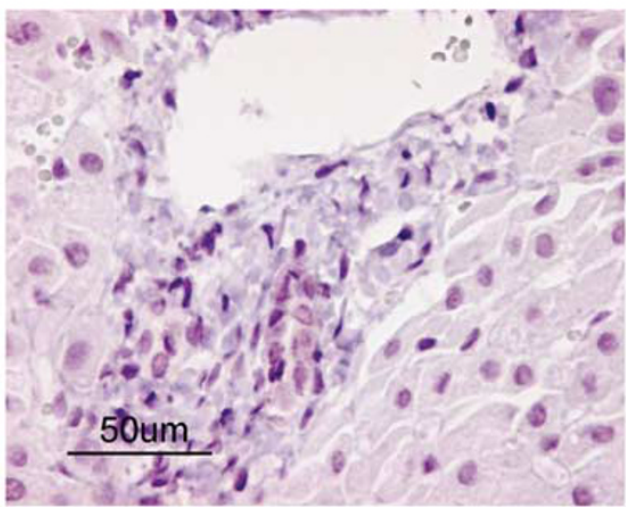

b

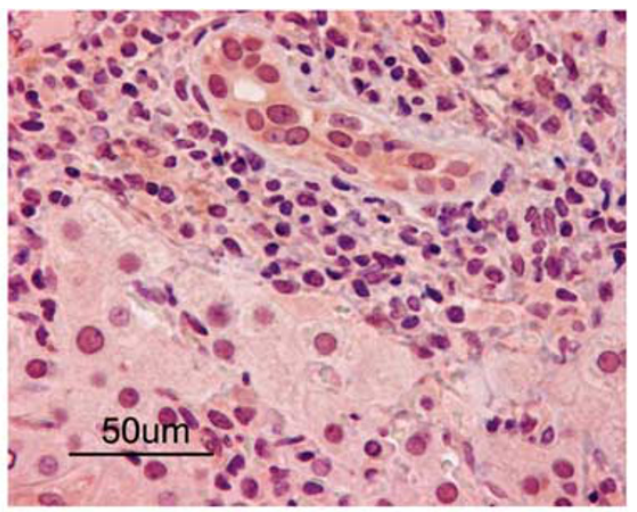

d
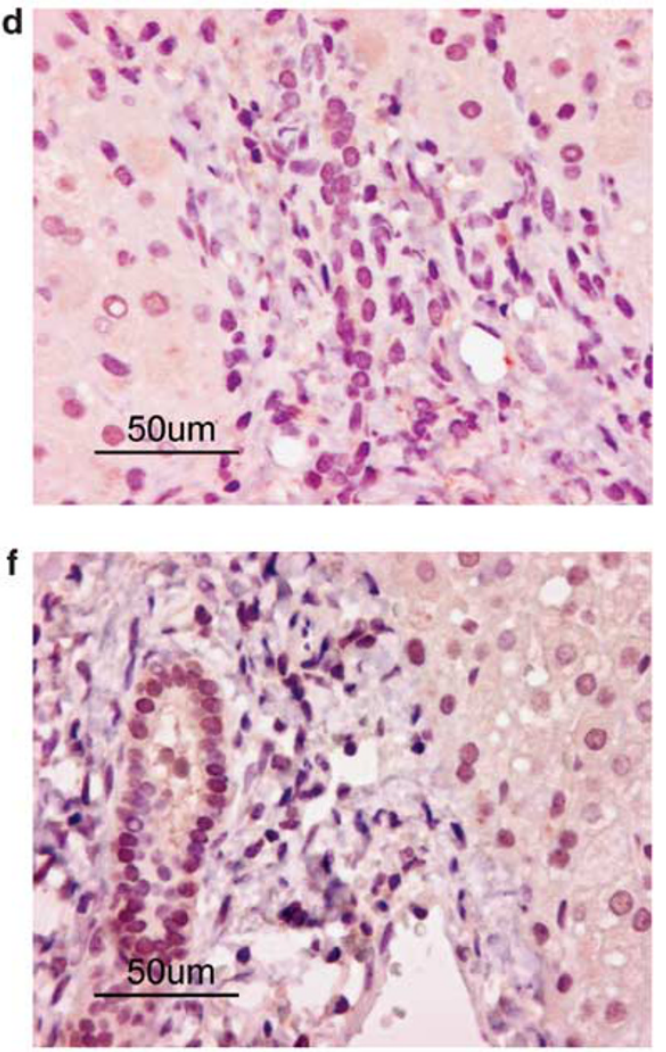

h

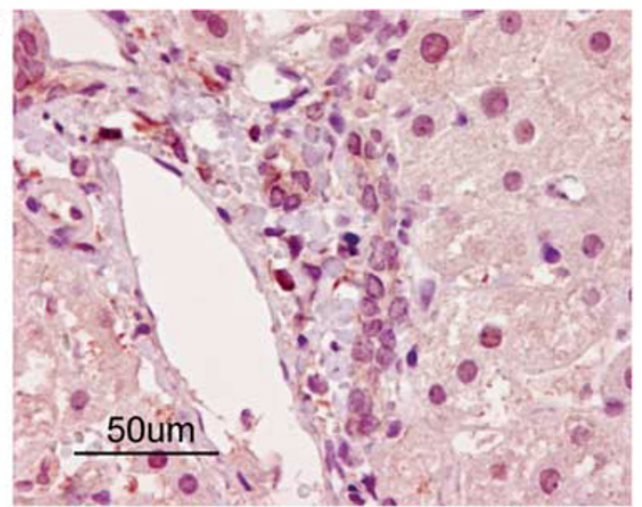

Figure 2 Four candidate molecules were evaluated for expression in the human liver specimen, stained with anti-cyclin-I (a, e), anti-Epac1 (b, f), anti-HSP70 $(\mathbf{c}, \mathbf{g})$ and anti-enolase-1 (d, h) ABs. (a-d) PBC liver and (e-h) normal liver. 
a

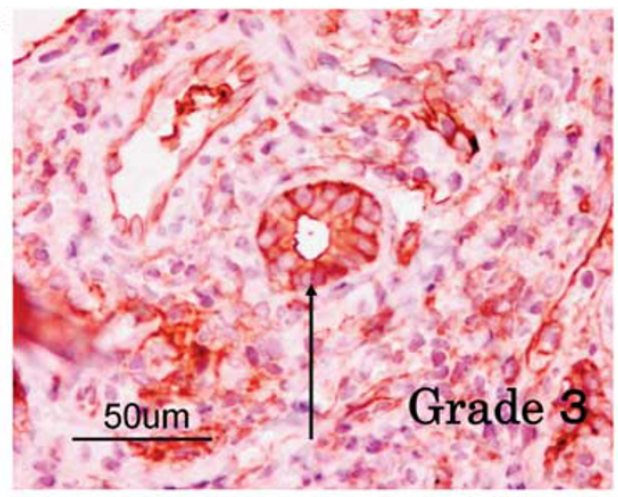

C

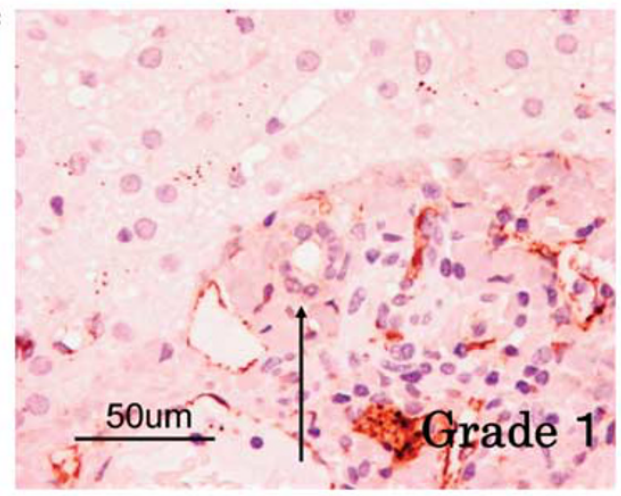

e

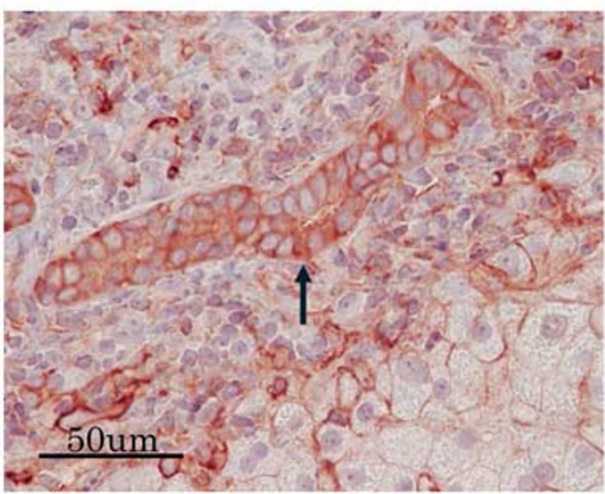

g

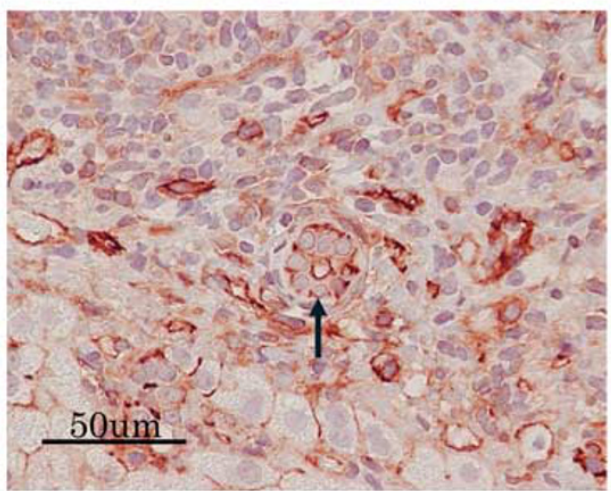

b

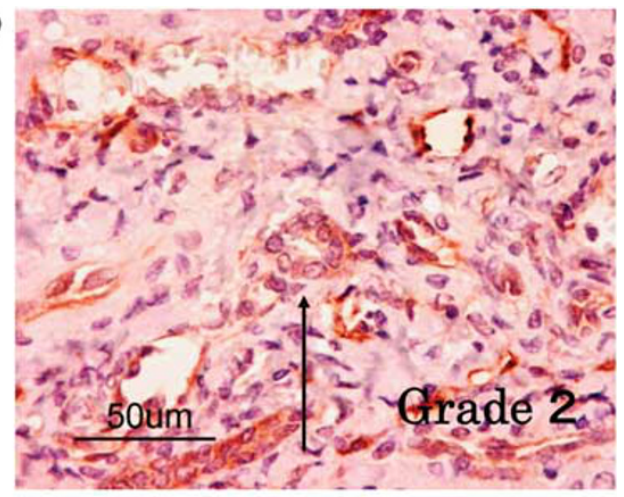

d

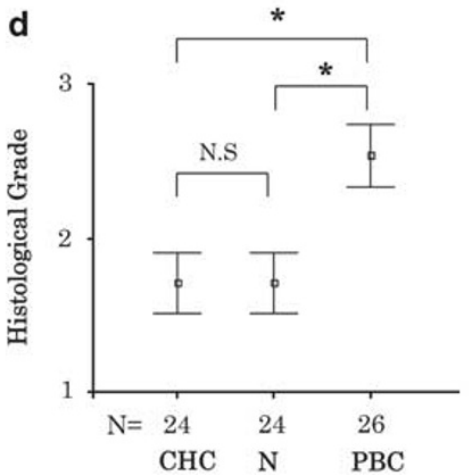

f

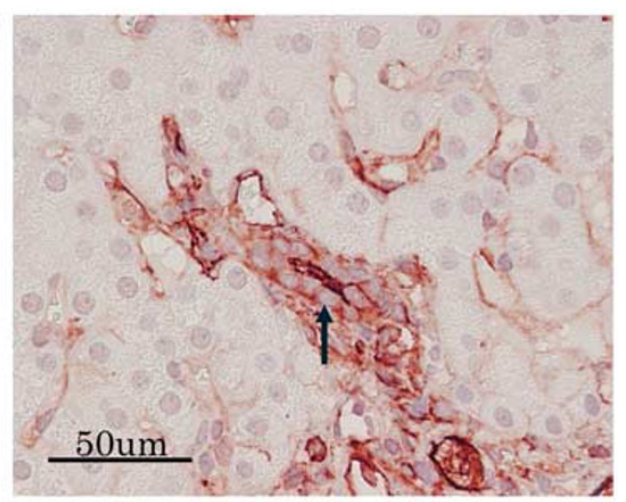

h
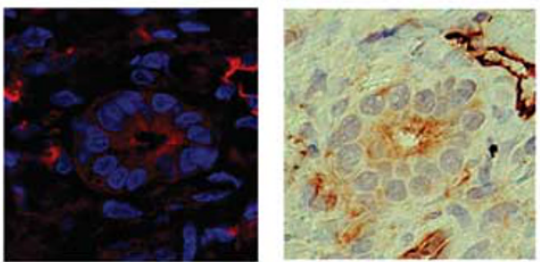

i
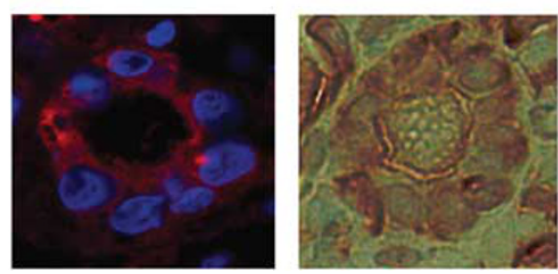

Figure 3 Human cholangiocytes expressed ANXA2. Representative images for the grading of expression of ANXA2 in cholangiocytes are shown ((a) grade 3, (b) grade 2 and (c) grade 1). Expression of ANXA2 was rather strong in the cholangiocytes of PBC liver as compared with that in the $\mathrm{CHC}$ or normal liver (d), according to the grading. Subcellular localization of ANXA2 was evaluated by immunohistochemistry in cholangiocytes of PBC livers. A representative staining pattern of cholangiocytes of PBC livers with predominant cytosolic (e) and membranous staining: apical (f) and basolateral staining (g) are shown. Immunofluorescence images also clearly demonstrate the subcellular localization of ANXA2 (h, i). Data sets were analyzed by Kruskal-Wallis test and Mann-Whitney U-test with Bonferroni correction; ${ }^{\star}=P<0.05$. 
Table 2 Correlation between clinical features and subcellular localization of ANXA2 in cholangiocytes

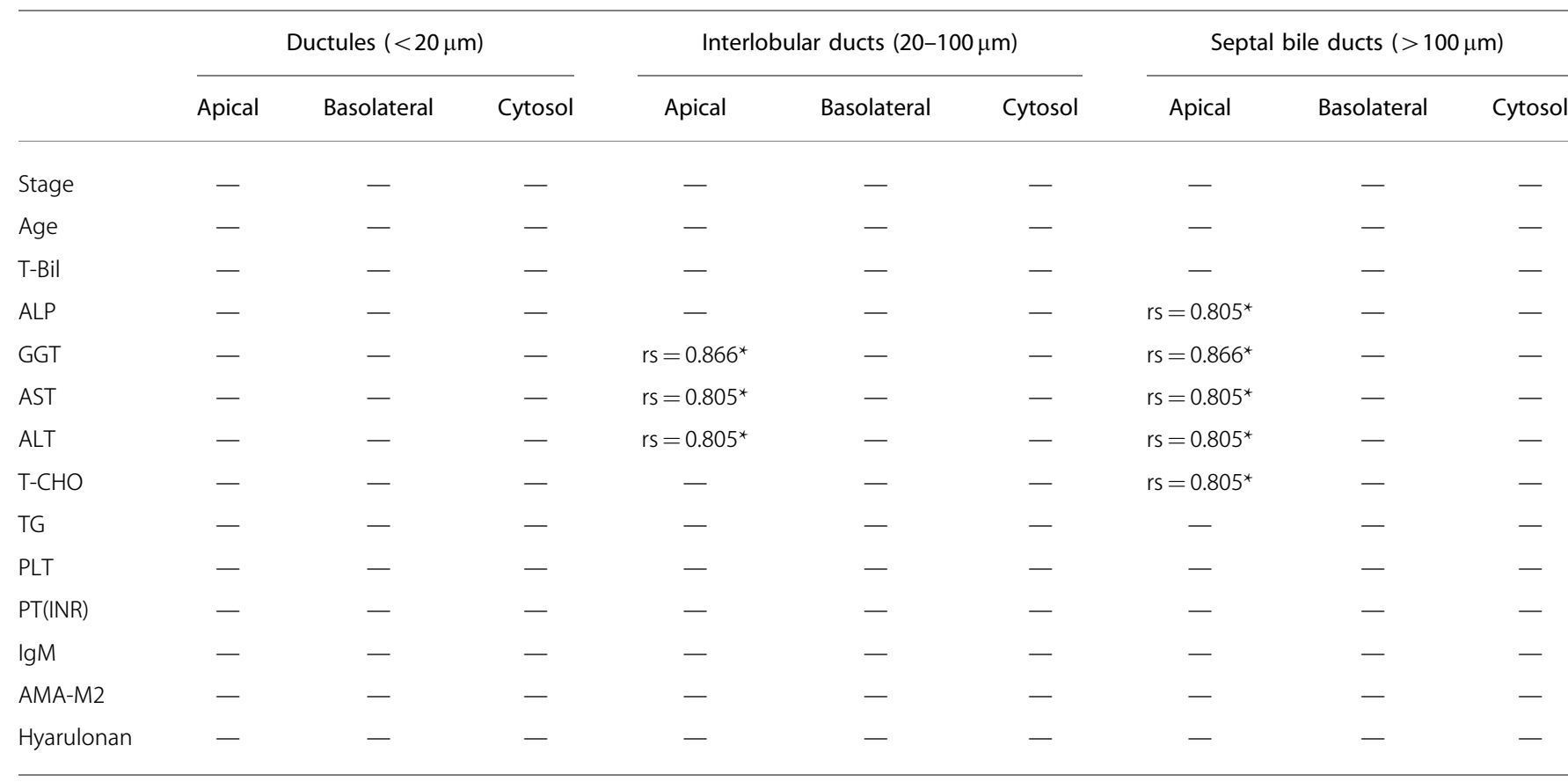

Abbreviations: - , not significant; ALP, alkaline phosphatase; ALT, alanine aminotransferase; AMA, anti mitochondria antibody; AST, aspartate aminotransferase; GGT, $\gamma$-glutamyl transferase; IgM, immunoglobulin M; PLT, platelet; PT, prothrombin time; T-Bil, serum concentration of total bilirubin; T-CHO, total cholesterol; TG, triglyceride.

Data sets were analyzed by Spearman's correlation.

${ }^{*} P$-values less than 0.05 were considered statistically significant. Ductules, interlobular ducts and septal bile ducts were defined as biliary ducts less than $20 \mu \mathrm{m}$, from 20 to $100 \mu \mathrm{m}$ and more than $100 \mu \mathrm{m}$ in diameter, respectively. Serum markers were measured by a usual method.

be involved in an $\mathrm{H}^{+}$-mediated mechanism. ${ }^{45}$ In addition, ANXA2 binds tissue plasminogen activator (tPA) as well as its substrate, plasminogen, and accelerates the catalytic efficiency of plasmin generation in vitro. ${ }^{46,47}$ Through conformational changes of ANXA2 that are caused by several factors, for example, binding of $\mathrm{Ca}^{2+}, \mathrm{pH}$ or the annexin itself, ANXA2 acquires high binding affinity to phospholipids, facilitating its relocation to the nuclear and plasma membranes. ANXA2 was discovered as an inhibitory molecule of phospholipase- $\mathrm{A} 2^{48}$ and recognized later as a major cellular substrate of the tyrosine kinase encoded by the SRC oncogene. Currently, various potential roles of this molecule have been proposed, which can be classified from two aspects, that is, unrelated intracellular and extracellular roles. Cell-surface ANXA2 functions as a co-receptor or platform for tPA that enables plasminogen to mediate the generation of plasmin, which leads to fibrinolysis, extracellular matrix degradation and cellular migration. ${ }^{49}$ In terms of alcoholic liver diseases, increased parenchymal expression of ANXA2 and its fibrinolytic role were proposed. ${ }^{13}$ We found expression of ANXA2 not only in the parenchymal hepatocytes of $\mathrm{PBC}$ and $\mathrm{CHC}$ liver, but also in the cholangioycytes of PBC. The subcellular localization of ANXA2 to the basolateral cell membrane of cholangiocytes or hepatocytes may be related to the fibrinolytic role of ANXA2 in the injured liver as a consequence of the activation of plasmin, leading to remodeling of the injured bile ducts. We speculated that the correlation between apical ANXA2 expression and serum cholestatic markers might indicate a unique role of this molecule in terms of the cholestasis or bile juice production of cholangiocytes. Bicarbonate secretion is one of the crucial roles of cholangiocytes, which is mediated by AE2 of the apical domain of cholangiocytes. Therefore, we focused on the putative role of ANXA2 in terms of bicarbonate secretion of the impaired cholangiocytes. There were several lines of indirect evidence that supported our hypothesis. ANXA2 is implicated in the exocytosis, endocytosis, organization of lipid-raft-like membrane constituents and ion-channel regulation. ${ }^{45}$ With regard to anion transport, ANXA2 serves as a $\mathrm{Cl}^{-}$channel regulator in vascular epithelial cells ${ }^{50}$ and as an essential molecule in the CFTR function in bronchial epithelial cells through formation of ANXA2/CFTR complex. ${ }^{51}$ The controlled induction of ANXA2 led to an unexpected increase of the $\mathrm{pH}_{\mathrm{i}}$ recovery that was DIDS-sensitive and was not affected by the CFTR inhibitor (data not shown). Moreover, induction of ANXA2 restored the AE activity impaired by IFN $\gamma$. Faint expression of AE2 in the cholangiocytes of the PBC livers was supported by the immunohistochemistry (data not shown), as mentioned before. ${ }^{16}$ Therefore, a compensatory role for the cholestasis of 
a
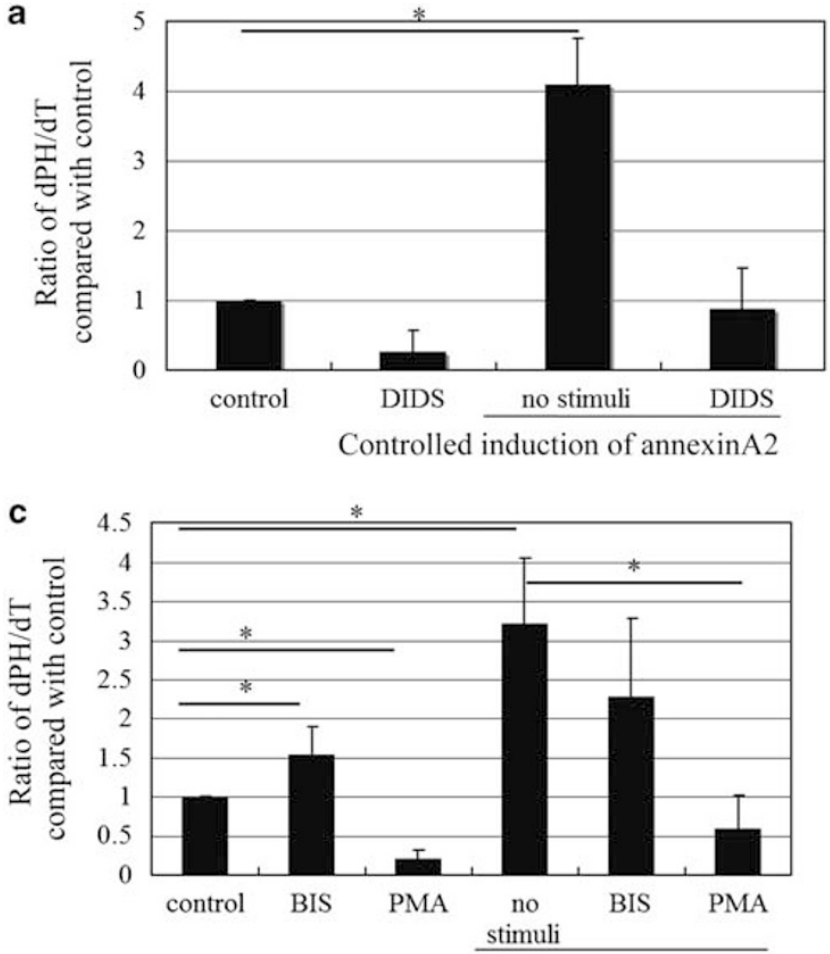

Controlled induction of annexinA2

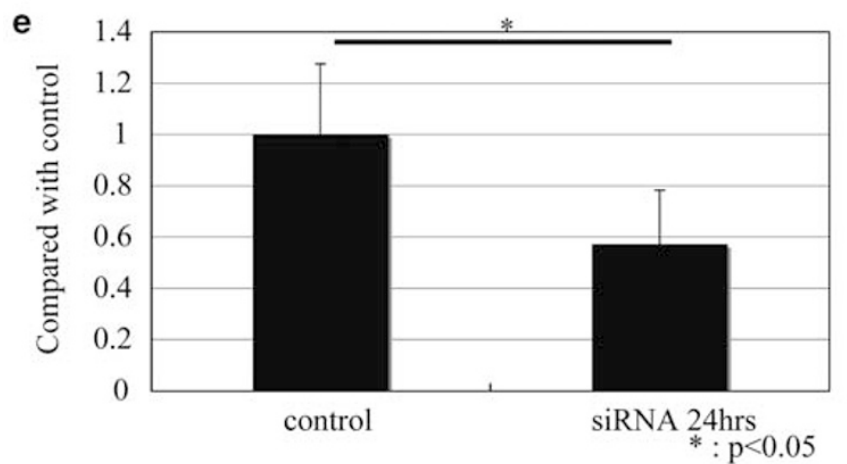

b

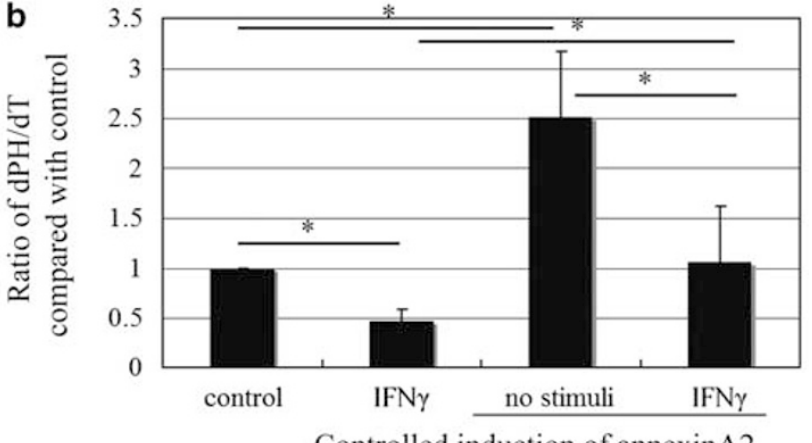

Controlled induction of annexinA2

d

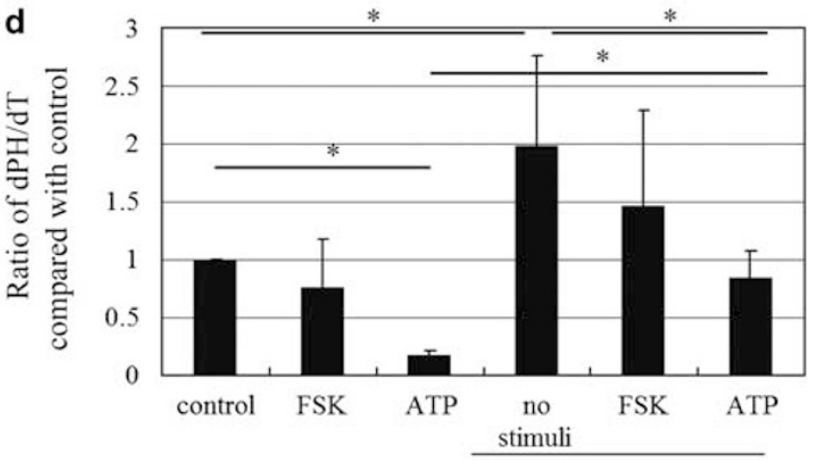

Controlled induction of annexinA2

f control siRNA $24 \mathrm{hrs}$

ANXA2

B-actin

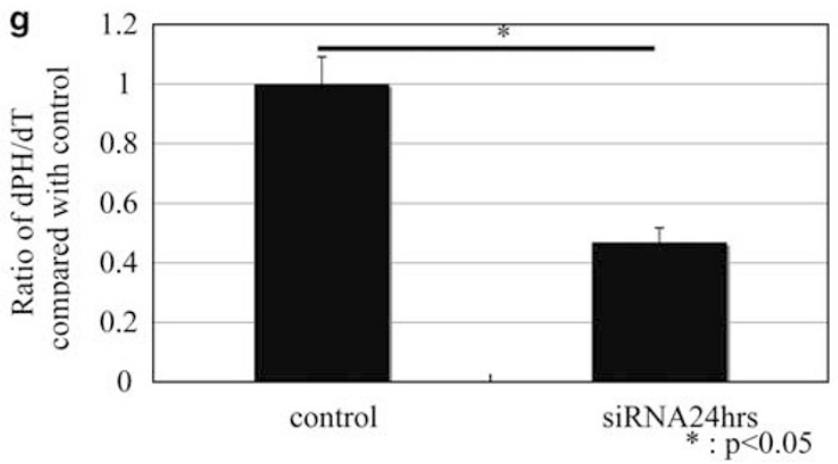

Figure 4 ANXA2 affected the AE activity of $\mathrm{H} 69$ cells. H69-anxa2 was established as described under section Materials and Methods. AE activity was assessed by measurement of the DIDS-sensitive $\mathrm{pH}_{\mathrm{i}}$ recovery from acute intracellular alkalization. The induction of ANXA2 upregulated the AE activity (a). Preincubation with IFN $\gamma$ at a concentration of $1000 \mathrm{IU} / \mathrm{ml}$ for $24 \mathrm{~h}$ caused downregulation of AE activity of $\mathrm{H} 69$ cells, which was partially recovered by induction of ANXA2 (b). PMA (950 nM), a pan-PKC activator, suppressed AE activity and $1 \mu \mathrm{M}$ of BIS, a pan-PKC inhibitor, increased AE activity significantly. The part of the AE activity augmented by induction of ANXA2 was negated by PMA (c). ATP, but not forskolin, attenuated the AE activity of H69 cells, which was partially rescued by controlled induction of ANXA2 (d). ANXA2 expression was significantly reduced to $46.9 \%$ as compared with that in the negative control by ANXA2 siRNA, as evaluated by immunoblotting and densitometry in triplicate $(\mathbf{e}, \mathbf{f}) . \mathrm{pH}_{\mathrm{i}}$ recovery was attenuated in the same manner by ANXA2 siRNA to $57.3 \%(\mathbf{g})$. Data sets were analyzed by Mann-Whitney $U$-test and $P$ values less than 0.05 were considered statistically significant; ${ }^{*}=P<0.05$. 

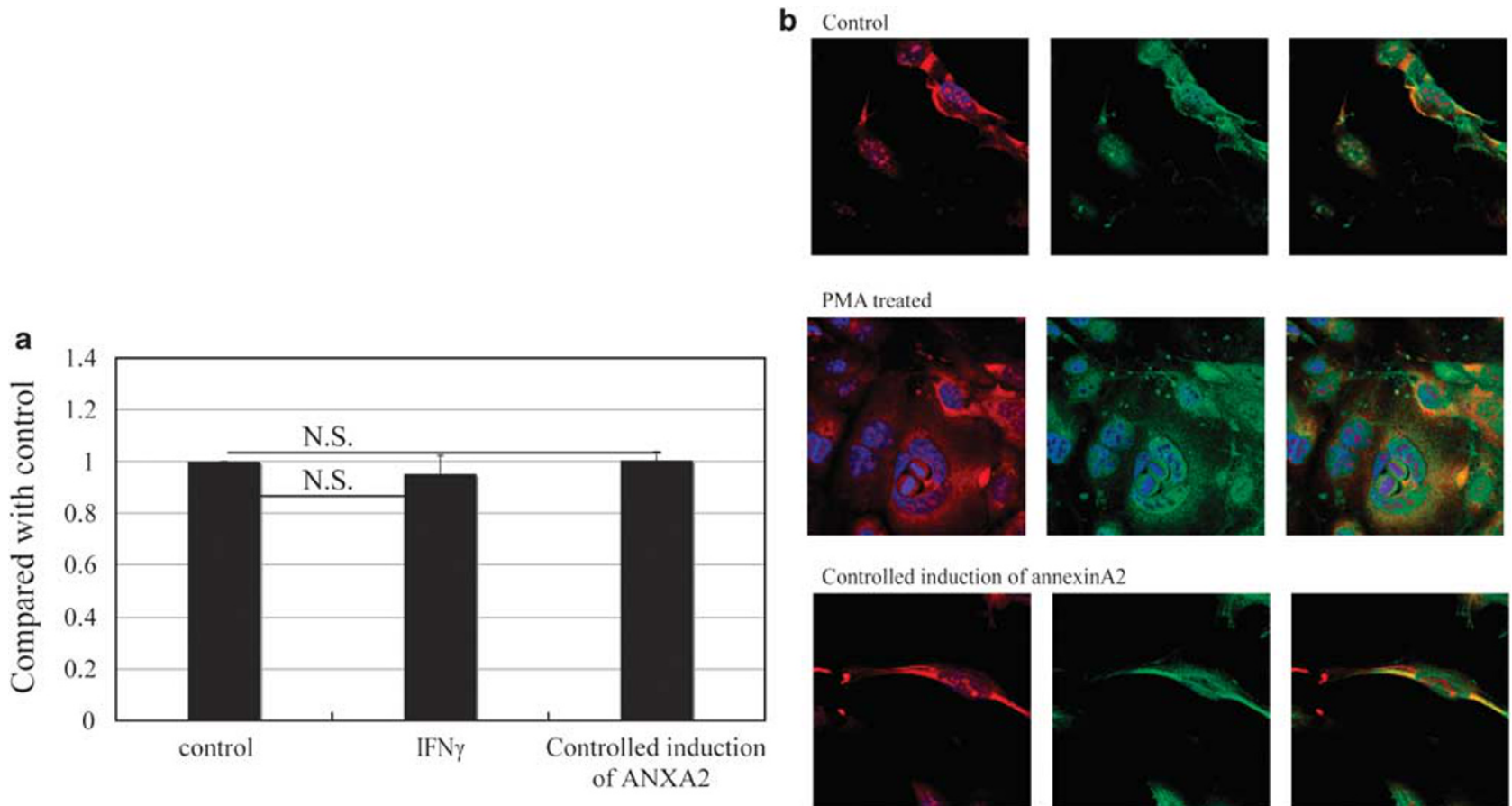

PMA treated
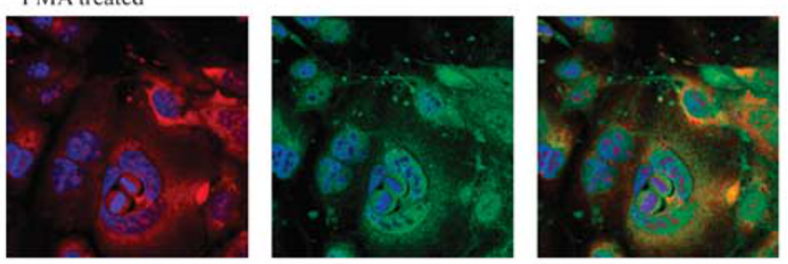

Controlled induction of annexinA2
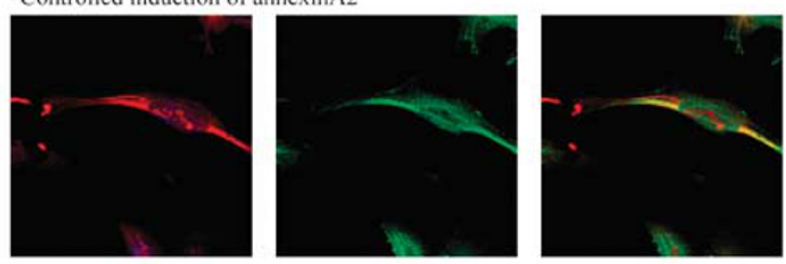

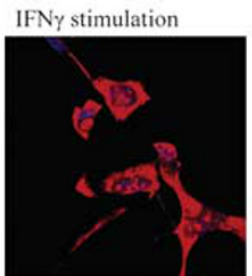

ANXA2

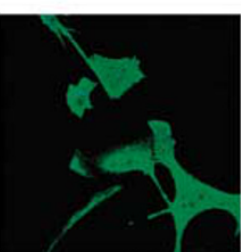

AE2

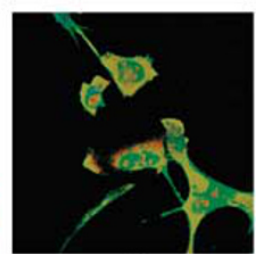

Merge

Figure 5 The AE2 status in $\mathrm{H} 69$ cells with the Tet-On vector of ANXA2 was confirmed by immunoblotting (a) and immunofluorescence (b). AE2 expression was not affected by IFN $\gamma$, which was constant even under DOX $(\mathbf{a}, \mathbf{b})$. Nuclear redistribution of ANXA2 from peripheral membrane and cytosol, or from cytosolic relocation of AE2 from peripheral membrane by PMA, is indicated by red and yellow arrows, respectively (b). Data sets were analyzed with MannWhitney $U$-test. NS = not significant.

ANXA2 was postulated for the bile formation through the modulation of the AE activity that colocalized to the apical membrane of cholangiocytes together with ANXA2.

Our investigation clarified, at least, some part of the underlying mechanism of the modulation of AE activity. PKC activator relocated ANXA2 and AE2 from the membrane compartment to the cytosol and nucleus in $\mathrm{H} 69$ cells, which would explain the mechanism of the AE activity attenuated by PKC activation. Methyl- $\beta$-cyclodextrin, which extracts cholesterol from cell membranes, ${ }^{52}$ totally abolished the $\mathrm{AE}$ activity regardless of the ANXA2 status (data not shown). Therefore, the AE activity was likely to be dependent on the microdomain, lipid rafts. AE2 that uptakes $\mathrm{Cl}^{-}$from the outer cell space and excretes $\mathrm{HCO}_{3}$ is a dimerized molecule that exists in the lipid rafts. ${ }^{53}$ Through modulation of the affinity of ANXA2 for the phospholipids under the existence of intracellular cytosolic $\mathrm{Ca}^{2+}$, the accessibility of ANXA2 to $\mathrm{AE}$ of the microdomain may become possible.
Extracellular ATP causes rapid increase of the intracellular $\mathrm{Ca}^{2+}$ concentration and subsequent activation of membrane $\mathrm{Cl}^{-}$channels through purinergic receptors in the process of bile formation by cholangiocytes. ${ }^{54-57}$ In the present study, we demonstrated the decrease of AE activity by the extracellular ATP and partial recovery of the AE activity by the induction of ANXA2. The phosphorylation status of the amino-acid residue of ANXA2 also modulates its binding affinity to the membrane. For example, the tyrosine phosphorylation of ANXA2 by pp60src decreases the binding of the protein to phospholipid vesicles at low $\mathrm{Ca}^{2+}$ concentrations, whereas serine phosphorylation of ANXA2 by PKC inhibits its ability to aggregate phospholipid vesicles. ${ }^{58}$ Extracellular ATP enhances both phosphorylation of 3-phosphoinositide-dependent protein kinase-1 (PDK1) and its translocation to the plasma membrane, through binding to P2Y receptors. ${ }^{59}$ Activated PDK1 by phosphorylation further maturates the downstream PKC by phosphorylation. 


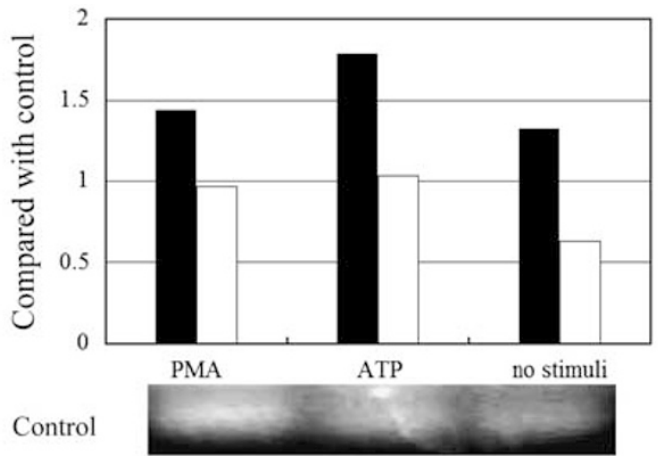

Controlled upregulation of annexin A2
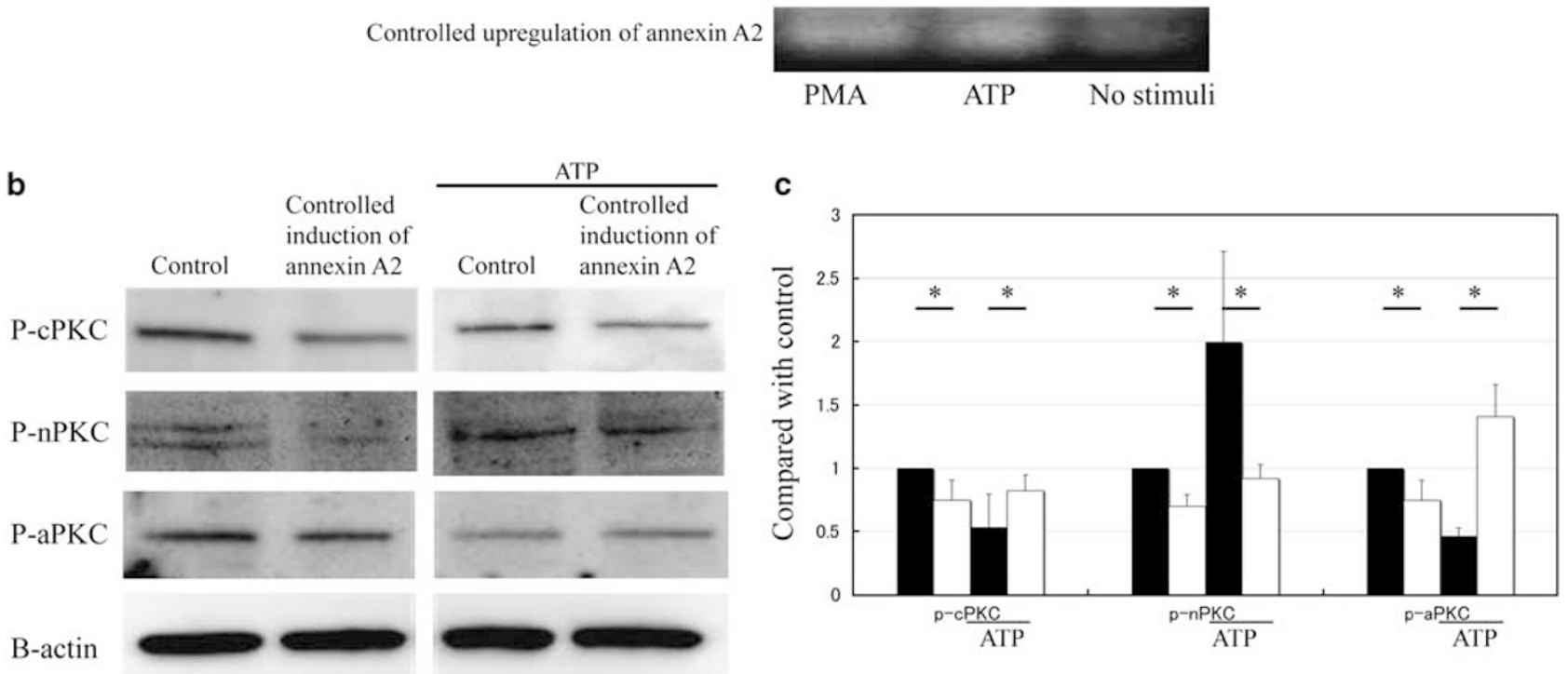

Figure 6 PMA and ATP increased the PKC activity of H69 cells shown as an increase of phospho-peptide, as evaluated by PepTag assay (a). Decreased induction of ANXA2 suppressed PKC activity. Phosphorylation of PKC subtypes as the maturated form evaluated by immunoblotting (b) and the results of densitometry (c) are shown. P-PKC $\alpha / \beta$ (as CPKC), p-PKC $\delta / \theta$ (as $\mathrm{nPKC}$ ), and $\mathrm{p}-\mathrm{PKC} \lambda / \zeta$ (as aPKC) all decreased in association with induction of ANXA2. Extracellular ATP increased the level of p-nPKC. ANXA2 decreased the level of p-nPKC and increased that of p-aPKC, paradoxically, together with ATP level. White bar, H69 lysates preincubated with DOX and black bar, without DOX. Data sets were analyzed with Mann-Whitney $U$-test. ${ }^{\star}=P<0.05$.

ANXA2 can bind directly and specifically to phosphatidylinositol-4,5-bisphosphate $\left(\operatorname{PtdIns}(4,5) \mathrm{P}_{2}\right)$, which is the source of diacylglycerol, a classical PKC activator, and inositol trisphosphate. ${ }^{60}$ With the PepTag Assay, we found that the ability of all PKC subtypes for phosphorylation of the substrate was attenuated by induction of ANXA2. The PKC activity might be directly modulated by the binding ability of ANXA2 to PtdIns $(4,5) \mathrm{P}_{2}$. The expressions of the maturated types of conventional, novel and atypical PKCs were all decreased by ANXA2. Extracellular ATP upregulated the maturated form of novel PKC, that is, subtype $\theta / \delta$, in contrast to conventional PKC, that is, subtype $\alpha / \beta$ (subtype $\gamma$ was not tested) and atypical PKC, that is, subtype $\lambda / \zeta$, which remained constant regardless of the supplementation of ATP. ANXA2 specifically suppressed the part of the maturated form of novel PKC that was increased by ATP. These findings indicate that ANXA2 could affect the maturation of whole PKC through modulating the phosphorylation status, which would affect the downstream signaling of cholangiocytes, including bicarbonate secretion.
The contradictive results of extracellular ATP on the AE activity, forskolin and CFTR inhibitor on the AE activity observed in the current study may be explained by the imbalance of the reaction caused by the extracellular ATP stimulation, such as the $\mathrm{Ca}^{2+}$ release from the ER, which leads to augmentation of $\mathrm{AE}$ activity and PKC maturation, which may counteract intracellular $\mathrm{Ca}^{2+}$ increase along with weak expression of CFTR in H69 cell speculated by the data mining of microarray (data not shown). Another possible weakness of our conclusion is that it was based on the results of the measurements of intracellular $\mathrm{pH}$, which might miss the actual bicarbonate secretion into the lumen by cholangiocytes. In the current study, we aimed at revealing the physiological interactions of molecules in human-derived samples under limited accessibility, which might also lead to results with certain limitations. Thus, we think that several lines of evidence related to AE2 activity should be reconciled for a better comprehension of biliary physiology and pathophysiology. 
In conclusion, ANXA2 expressed in the biliary epithelial cells might be a histological marker of cholangiopathy, including PBC. ANXA2 would have a compensatory role for bicarbonate secretion through modulating the $\mathrm{AE}$ activity of human cholangiocytes under pathophysiological conditions.

\section{ACKNOWLEDGEMENTS}

Grant support: Supported in part by Health and Labour Sciences Research Grants for the Research on Measures for Intractable Diseases (from the Ministry of Health, Labour and Welfare of Japan), from Grant-in-Aid for Scientific Research C (21590822 to YU and 20590755 to KF) from the Japanese Society of Promotion of Science (JSPS) and from National Institutes of Health Grants DK074768 and P30 DK-34928 to DMJ.

\section{DISCLOSURE/CONFLICT OF INTEREST}

The authors declare no conflict of interest.

1. Harada K, Isse K, Nakanuma Y. Interferon gamma accelerates NF-kappaB activation of biliary epithelial cells induced by Toll-like receptor and ligand interaction. J Clin Pathol 2006;59:184-190.

2. Yasoshima $\mathrm{M}$, Kono $\mathrm{N}$, Sugawara $\mathrm{H}$, et al. Increased expression of interleukin- 6 and tumor necrosis factor-alpha in pathologic biliary epithelial cells: in situ and culture study. Lab Invest 1998;78:89-100.

3. Berg PA, Klein R, Rocken M. Cytokines in primary biliary cirrhosis. Semin Liver Dis 1997;17:115-123.

4. Harada K, Van de Water J, Leung PS, et al. In situ nucleic acid hybridization of cytokines in primary biliary cirrhosis: predominance of the Th1 subset. Hepatology 1997;25:791-796.

5. Spirli C, Nathanson MH, Fiorotto R, et al. Proinflammatory cytokines inhibit secretion in rat bile duct epithelium. Gastroenterology 2001;121:156-169.

6. Mayran N, Parton RG, Gruenberg J. Annexin II regulates multivesicular endosome biogenesis in the degradation pathway of animal cells. EMBO J 2003;22:3242-3253.

7. Gruenberg J, Stenmark H. The biogenesis of multivesicular endosomes. Nat Rev Mol Cell Biol 2004;5:317-323.

8. Zobiack N, Rescher U, Ludwig C, et al. The annexin 2/S100A10 complex controls the distribution of transferrin receptor-containing recycling endosomes. Mol Biol Cell 2003;14:4896-4908.

9. Harder T, Kellner R, Parton RG, et al. Specific release of membranebound annexin II and cortical cytoskeletal elements by sequestration of membrane cholesterol. Mol Biol Cell 1997;8:533-545.

10. Zeuschner D, Stoorvogel W, Gerke V. Association of annexin 2 with recycling endosomes requires either calcium- or cholesterol-stabilized membrane domains. Eur J Cell Biol 2001;80:499-507.

11. Rescher U, Gerke V. Annexins-unique membrane binding proteins with diverse functions. J Cell Sci 2004;117:2631-2639.

12. Moss SE, Morgan RO. The annexins. Genome Biol 2004;5:219.

13. Seth D, Hogg PJ, Gorrell MD, et al. Direct effects of alcohol on hepatic fibrinolytic balance: implications for alcoholic liver disease. J Hepatol 2008;48:614-627.

14. Alper SL. Molecular physiology of SLC4 anion exchangers. Exp Physiol 2006;91:153-161.

15. Banales JM, Arenas F, Rodriguez-Ortigosa CM, et al. Bicarbonate-rich choleresis induced by secretin in normal rat is taurocholate-dependent and involves AE2 anion exchanger. Hepatology 2006;43:266-275.

16. Medina JF, Martinez A, Vazquez JJ, et al. Decreased anion exchanger 2 immunoreactivity in the liver of patients with primary biliary cirrhosis. Hepatology 1997;25:12-17.

17. Salas JT, Banales JM, Sarvide $S$, et al. Ae2a, b-deficient mice develop antimitochondrial antibodies and other features resembling primary biliary cirrhosis. Gastroenterology 2008;134:1482-1493.

18. Grubman SA, Perrone RD, Lee DW, et al. Regulation of intracellular pH by immortalized human intrahepatic biliary epithelial cell lines. Am J Physiol 1994;266:G1060-G1070.

19. Scheuer P. Primary biliary cirrhosis. Proc R Soc Med 1967;60: $1257-1260$.

20. Inoue J, Takahashi M, Nishizawa T, et al. High prevalence of hepatitis delta virus infection detectable by enzyme immunoassay among apparently healthy individuals in Mongolia. J Med Virol 2005;76: 333-340.

21. Spirli C, Granato A, Zsembery $\mathrm{K}$, et al. Functional polarity of $\mathrm{Na}+\mathrm{H}+$ and $\mathrm{Cl}-/ \mathrm{HCO}-$ exchangers in a rat cholangiocyte cell line. Am J Physiol 1998;275:G1236-G1245.

22. Martinez-Anso E, Castillo JE, Diez J, et al. Immunohistochemical detection of chloride/bicarbonate anion exchangers in human liver. Hepatology 1994;19:1400-1406.

23. Hassan HA, Mentone S, Karniski LP, et al. Regulation of anion exchanger Slc26a6 by protein kinase C. Am J Physiol Cell Physiol 2007;292:C1485-C1492.

24. Han J, Lee $\mathrm{Y}$, Yeom $\mathrm{KH}$, et al. The Drosha-DGCR8 complex in primary microRNA processing. Genes Dev 2004;18:3016-3027.

25. Rubin BY, Anderson SL, Xing L, et al. Interferon induces tryptophanyltRNA synthetase expression in human fibroblasts. J Biol Chem 1991;266:24245-24248.

26. de Weille JR, Bassilana F, Lazdunski $M$, et al. Identification, functional expression and chromosomal localisation of a sustained human proton-gated cation channel. FEBS Lett 1998;433:257-260.

27. Quelle FW, Shimoda K, Thierfelder W, et al. Cloning of murine Stat6 and human Stat6, Stat proteins that are tyrosine phosphorylated in responses to IL- 4 and IL-3 but are not required for mitogenesis. Mol Cell Biol 1995;15:3336-3343.

28. Katoh M. Molecular cloning and characterization of human WINS1 and mouse Wins2, homologous to Drosophila segment polarity gene Lines (Lin). Int J Mol Med 2002;10:155-159.

29. Lindvall JM, Blomberg KE, Valiaho J, et al. Bruton's tyrosine kinase: cell biology, sequence conservation, mutation spectrum, siRNA modifications, and expression profiling. Immunol Rev 2005;203: 200-215.

30. Leimeister C, Steidl C, Schumacher N, et al. Developmental expression and biochemical characterization of Emu family members. Dev Biol 2002;249:204-218.

31. Izumikawa $T$, Koike $T$, Shiozawa $S$, et al. Identification of chondroitin sulfate glucuronyltransferase as chondroitin synthase-3 involved in chondroitin polymerization: chondroitin polymerization is achieved by multiple enzyme complexes consisting of chondroitin synthase family members. J Biol Chem 2008;283:11396-11406.

32. Nakamura T, Sanokawa R, Sasaki YF, et al. Cyclin I: a new cyclin encoded by a gene isolated from human brain. Exp Cell Res 1995;221:534-542.

33. Gruber $\mathrm{AD}$, Elble $\mathrm{RC}$, Ji HL, et al. Genomic cloning, molecular characterization, and functional analysis of human CLCA1, the first human member of the family of $\mathrm{Ca} 2+$-activated $\mathrm{Cl}$ - channel proteins. Genomics 1998;54:200-214.

34. Honore $\mathrm{B}$, Rasmussen $\mathrm{HH}$, Vorum $\mathrm{H}$, et al. Heterogeneous nuclear ribonucleoproteins $\mathrm{H}, \mathrm{H}^{\prime}$, and $\mathrm{F}$ are members of a ubiquitously expressed subfamily of related but distinct proteins encoded by genes mapping to different chromosomes. J Biol Chem 1995;270: 28780-28789.

35. Weis WI, Taylor ME, Drickamer K. The C-type lectin superfamily in the immune system. Immunol Rev 1998;163:19-34.

36. Arya R, Lalloz MR, Bellingham AJ, et al. Evidence for founder effect of the Glu104Asp substitution and identification of new mutations in triosephosphate isomerase deficiency. Hum Mutat 1997; 10:290-294.

37. Yoshikawa T, Padigaru M, Karkera JD, et al. Genomic structure and novel variants of myo-inositol monophosphatase 2 (IMPA2). Mol Psychiatry 2000;5:165-171.

38. Pelham HR. Speculations on the functions of the major heat shock and glucose-regulated proteins. Cell 1986;46:959-961.

39. Neesen J, Koehler MR, Kirschner R, et al. Identification of dynein heavy chain genes expressed in human and mouse testis: chromosomal localization of an axonemal dynein gene. Gene 1997; 200:193-202.

40. Tietz PS, McNiven MA, Splinter PL, et al. Cytoskeletal and motor proteins facilitate trafficking of AQP1-containing vesicles in cholangiocytes. Biol Cell 2006;98:43-52.

41. de Rooij J, Zwartkruis FJ, Verheijen MH, et al. Epac is a Rap1 guaninenucleotide-exchange factor directly activated by cyclic AMP. Nature 1998;396:474-477.

42. Lopez-Alemany R, Suelves M, Diaz-Ramos A, et al. Alpha-enolase plasminogen receptor in myogenesis. Front Biosci 2005;10:30-36. 
43. Akisawa N, Maeda T, Iwasaki S, et al. Identification of an autoantibody against alpha-enolase in primary biliary cirrhosis. J Hepatol 1997:26:845-851.

44. Camors E, Monceau V, Charlemagne D. Annexins and Ca2+ handling in the heart. Cardiovasc Res 2005;65:793-802.

45. Gerke V, Creutz CE, Moss SE. Annexins: linking Ca2+ signalling to membrane dynamics. Nat Rev Mol Cell Biol 2005;6:449-461.

46. Hajjar KA, Jacovina AT, Chacko J. An endothelial cell receptor for plasminogen/tissue plasminogen activator. I. Identity with annexin II. J Biol Chem 1994;269:21191-21197.

47. Kassam G, Choi KS, Ghuman J, et al. The role of annexin II tetramer in the activation of plasminogen. J Biol Chem 1998;273:4790-4799.

48. Huang KS, Wallner BP, Mattaliano RJ, et al. Two human 35 kDa inhibitors of phospholipase A2 are related to substrates of pp60v-src and of the epidermal growth factor receptor/kinase. Cell 1986;46:191-199.

49. Kim J, Hajjar KA. Annexin II: a plasminogen-plasminogen activator co-receptor. Front Biosci 2002;7:d341-d348.

50. Nilius B, Gerke V, Prenen J, et al. Annexin II modulates volumeactivated chloride currents in vascular endothelial cells. J Biol Chem 1996;271:30631-30636.

51. Borthwick LA, McGaw J, Conner G, et al. The formation of the CAMP/ protein kinase A-dependent annexin 2-S100A10 complex with cystic fibrosis conductance regulator protein (CFTR) regulates CFTR channel function. Mol Biol Cell 2007;18:3388-3397.

52. Zidovetzki R, Levitan I. Use of cyclodextrins to manipulate plasma membrane cholesterol content: evidence, misconceptions and control strategies. Biochim Biophys Acta 2007;1768:1311-1324.
53. Banales JM, Prieto J, Medina JF. Cholangiocyte anion exchange and biliary bicarbonate excretion. World J Gastroenterol 2006;12: 3496-3511.

54. Woo K, Dutta AK, Patel V, et al. Fluid flow induces mechanosensitive ATP release, calcium signalling and $\mathrm{Cl}-$ transport in biliary epithelial cells through a PKCzeta-dependent pathway. J Physiol 2008;586: 2779-2798.

55. Hwang TH, Schwiebert EM, Guggino WB. Apical and basolateral ATP stimulates tracheal epithelial chloride secretion via multiple purinergic receptors. Am J Physiol 1996;270: C1611-C1623.

56. Bodily $\mathrm{K}$, Wang $\mathrm{Y}$, Roman $\mathrm{R}$, et al. Characterization of a swellingactivated anion conductance in homozygous typing cell hepatoma cells. Hepatology 1997;25:403-410.

57. Higuchi S, Nakasako M, Kudo T. Crystallization and preliminary X-ray diffraction studies of hyperthermostable glutamate dehydrogenase from Thermococcus profundus. Acta Crystallogr D Biol Crystallogr 1999;55:1917-1919.

58. Johnstone SA, Hubaishy I, Waisman DM. Phosphorylation of annexin II tetramer by protein kinase $C$ inhibits aggregation of lipid vesicles by the protein. J Biol Chem 1992;267:25976-25981.

59. Montiel $M$, de la Blanca EP, Jimenez E. P2Y receptors activate MAPK/ERK through a pathway involving PI3K/PDK1/PKC-zeta in human vein endothelial cells. Cell Physiol Biochem 2006;18: 123-134.

60. Gerke V, Moss SE. Annexins: from structure to function. Physiol Rev $2002 ; 82 \cdot 331-371$. 